PROCEEDINGS OF THE

AMERICAN MATHEMATICAL SOCIETY

Volume 139, Number 12, December 2011, Pages 4181-4192

S 0002-9939(2011)11204-8

Article electronically published on July 7, 2011

\title{
STAR CONFIGURATION POINTS AND GENERIC PLANE CURVES
}

\author{
ENRICO CARLINI AND ADAM VAN TUYL
}

(Communicated by Irena Peeva)

\begin{abstract}
Let $\ell_{1}, \ldots, \ell_{l}$ be $l$ lines in $\mathbb{P}^{2}$ such that no three lines meet in a point. Let $\mathbb{X}(l)$ be the set of points $\left\{\ell_{i} \cap \ell_{j} \mid 1 \leq i<j \leq l\right\} \subseteq \mathbb{P}^{2}$. We call $\mathbb{X}(l)$ a star configuration. We describe all pairs $(d, l)$ such that the generic degree $d$ curve in $\mathbb{P}^{2}$ contains an $\mathbb{X}(l)$. Our proof strategy uses both a theoretical and an explicit algorithmic approach. We also describe how one may extend our algorithmic approach to similar problems.
\end{abstract}

\section{INTRODUCTION}

The problem of studying subvarieties of algebraic varieties is a crucial one in algebraic geometry. Consider, for example, the crucial case of divisors. The study of subvarieties of hypersurfaces in $\mathbb{P}^{n}$ has a particularly rich history. For example, one can look for the existence of $m$-dimensional linear spaces on generic hypersurfaces of degree $d$ leading to the theory of Fano varieties and to the well-known formula relating $n, m$ and $d$ (e.g., see [10, Theorem 12.8]).

Because linear spaces are complete intersections, it is natural to look for the existence of complete intersection subvarieties on a generic hypersurface. The case of codimension two complete intersections was first studied by Severi [15], and later generalized and extended to higher codimensions by Noether, Lefschetz [11] and Groethendieck [9]. Recently, in [3], secant varieties were used to give a complete solution for the existence of complete intersections of codimension $r$ on generic hypersurfaces in $\mathbb{P}^{n}$ when $2 r \leq n+2$. Fewer results are known if the codimension of the complete intersection is large, i.e., the codimension is close to the dimension of the ambient space. In [16 the case of complete intersection curves is studied and completely solved. The case of complete intersection points on generic surfaces in $\mathbb{P}^{3}$ is considered in 4 where some asymptotic results are presented. The case of complete intersection points in $\mathbb{P}^{2}$ is a special case of [3], and it is completely solved.

Taking our inspiration from [3, 4, we examine the problem of determining when special configurations of points lie on a generic degree $d$ plane curve in $\mathbb{P}^{2}$. We shall focus on star configurations of points. Consider $l$ lines in the plane $\ell_{1}, \ldots, \ell_{l} \subset \mathbb{P}^{2}$ such that $\ell_{i} \cap \ell_{j} \cap \ell_{k}=\emptyset$. The set of points $\mathbb{X}(l)$ consisting of the $\left(\begin{array}{l}l \\ 2\end{array}\right)$ pairwise

Received by the editors October 13, 2010.

2010 Mathematics Subject Classification. Primary 14M05, 14H50.

Key words and phrases. Star configurations, generic plane curves.

(C)2011 American Mathematical Society

Reverts to public domain 28 years from publication 
intersections of the lines $\ell_{i}$ is called a star configuration. In this paper, we address the following question:

Question 1.1. For what pairs $(d, l)$ does the generic degree $d$ plane curve contain a star configuration $\mathbb{X}(l) \subseteq \mathbb{P}^{2}$ ?

The name star configuration was suggested by A. V. Geramita, since $\mathbb{X}(5)$ is the ten points of intersections when drawing a star using five lines (see Figure 4.2.4 in [6]). The configurations $\mathbb{X}(l)$ appeared in the work of Geramita, Migliore, and Sabourin 8 as the support of a set of double points whose Hilbert function exhibited an extremal behaviour. More recently, Cooper, Harbourne, and Teitler [6] computed the Hilbert function of any homogeneous set of fat points supported on $\mathbb{X}(l)$. Bocci and Harbourne [1] used star configurations (and their generalizations) to compare the symbolic and regular powers of an ideal. Further properties of star configurations continue to be uncovered, e.g., ongoing work of Geramita, Harbourne, Migliore [7.

We can answer Question 1.1 because we can exploit the rich algebraic structure of star configurations. In particular, we will require the fact that one can easily write down a list of generators for $I_{\mathbb{X}(l)}$, the defining ideal of $\mathbb{X}(l)$, as well as the fact that the Hilbert function of $\mathbb{X}(l)$ is the same as the Hilbert function of $\left(\begin{array}{l}l \\ 2\end{array}\right)$ generic points in $\mathbb{P}^{2}$. Using these properties, among others, we give the following solution to Question 1.1, repeating Theorem 6.3 from Section 6:

Theorem 6.3. Let $l \geq 2$. Then the generic degree $d$ plane curve contains a star configuration $\mathbb{X}(l)$ if and only if

(i) $l=2$ and $d \geq 1$ or

(ii) $l=3$ and $d \geq 2$ or

(iii) $l=4$ and $d \geq 3$ or

(iv) $l=5$ and $d \geq 5$.

Our proof is broken down into a number of cases. It will follow directly from the generators of $I_{\mathbb{X}(l)}$ that Question [1.1 can have no solution for $d<l-1$. Using a simple dimension-counting argument, Theorem 3.1 shows that for $l \geq 6$, there is no solution to Question 1.1. The cases $l=2$ and $l=3$ are trivial cases, so the bulk of the paper will be devoted to the cases $l=4$ and $l=5$.

To prove these cases, we rephrase Question 1.1 into a purely ideal theoretic question (see Lemma 4.3). Precisely, we construct a new ideal $I$ from the generators of $I_{\mathbb{X}(l)}$ and the linear forms defining the lines $\ell_{1}, \ldots, \ell_{l}$. We then show that Question $\left[1.1\right.$ is equivalent to determining whether $I_{d}=(\mathbb{C}[x, y, z])_{d}$. We then answer this new algebraic reformulation. We wish to stress that this reformulation is particularly interesting in that it allows for explicit computations using a computer algebra system (see Remark 4.4 and Example 4.5). In Section 7 we reformulate our approach into a general principle which allows for an algorithmic approach to similar problems.

The proofs for the cases $(d, l)=(3,4)$ and $(4,5)$ are, we believe, especially interesting. To prove that Question 1.1 is true for $(3,4)$, we exploit the natural group structure of the plane cubic curve to find an $\mathbb{X}(4)$ on the curve. To show the non-existence of a solution for $(4,5)$, we require the classical theory of Lüroth quartics. Lüroth quartics are the plane quartics that pass through an $\mathbb{X}(5)$. Lüroth quartics have the property of forming a hypersurface in the space of plane quartics. 
The existence of this hypersurface is the obstruction for the existence of a solution when $(d, l)=(4,5)$.

Our paper is structured as follows. In Section 2, we describe the needed algebraic properties of star configurations. In Section 3, we give some asymptotic results. In Section 4, in preparation for the last two sections, we rephrase Question 1.1 into an equivalent algebraic problem. Sections 5 and 6 deal with the cases $l=4$ and $l=5$, respectively. The final section concludes with additional remarks, among which is an alternative proof strategy using geometry.

\section{Star configurations}

Throughout this paper, we set $S=\mathbb{C}[x, y, z]$ and we denote its $d$ th homogeneous piece with $S_{d}$. Moreover, we fix standard monomial bases in each $S_{d}$ in such a way that $\mathbb{P} S_{d}$ will be identified with $\mathbb{P}^{N_{d}}$ where $N_{d}=\left(\begin{array}{c}d+2 \\ 2\end{array}\right)-1$. We recall the relevant definitions concerning star configurations of points in $\mathbb{P}^{2}$.

Let $l \geq 2$ be an integer. The scheme $\mathbb{X}(l) \subset \mathbb{P}^{2}$ is said to be a star configuration if it consists of $\left(\begin{array}{l}l \\ 2\end{array}\right)$ distinct points which are the pairwise intersections of $l$ distinct lines, say $\ell_{1}, \ldots, \ell_{l}$, where no three lines pass through the same point. We will also call $\mathbb{X}(l)$ a star configuration set of points.

Note that when $l=2$, then $\mathbb{X}(2)$ consists of a single point. When $l=3$, then $\mathbb{X}(3)$ is any set of three points in $\mathbb{P}^{2}$, provided the three points do not lie on the same line. It is clear that any degree $d \geq 1$ plane curve contains a point. Furthermore, when $d \geq 2$, the generic degree $d$ plane curve will contain three points not lying on a line. These remarks take care of the trivial cases of Theorem 6.3, which we summarize as a lemma:

Lemma 2.1. The generic degree $d$ plane curve contains a star configuration $\mathbb{X}(l)$ with $l=2$, respectively $l=3$, if and only if $d \geq 1$, respectively $d \geq 2$.

Given an $\mathbb{X}(l)$, for each $i=1, \ldots, l$, we let $L_{i}$ denote a linear form in $S_{1}$ defining the line $\ell_{i}$. The defining ideal of $I_{\mathbb{X}(l)}$ is then given by

$$
I_{\mathbb{X}(l)}=\bigcap_{i \neq j}\left(L_{i}, L_{j}\right) .
$$

We will sometimes write a point of $\mathbb{X}(l)$ as $p_{i, j}$, where $p_{i, j}$ is the point defined by the ideal $\left(L_{i}, L_{j}\right)$; i.e., $p_{i, j}$ is the point of intersection of the lines $\ell_{i}$ and $\ell_{j}$. The following lemma describes the generators of $I_{\mathbb{X}(l)}$; we will exploit this fact throughout the paper.

Lemma 2.2. Let $l \geq 2$, and let $\mathbb{X}(l)$ denote the star configuration constructed from the lines $\ell_{1}, \ldots, \ell_{l}$. If $L_{i}$ is a linear form defining $\ell_{i}$ for $i=1, \ldots, l$, then

$$
I_{\mathbb{X}(l)}=\left(\hat{L}_{1}, \ldots, \hat{L}_{l}\right) \text { where } \hat{L}_{i}=\prod_{j \neq i} L_{j} .
$$

For a proof of this fact, see [2, Claim in Proposition 3.4]. From this description of the generators, we see that $I_{\mathbb{X}(l)}$ is generated in degree $l-1$. Thus, for $d<l-1$, there are no plane curves of degree $d$ that contain an $\mathbb{X}(l)$ since $\left(I_{\mathbb{X}(l)}\right)_{d}=(0)$. Hence, as a corollary, we get some partial information about Question 1.1 . 
Corollary 2.3. If $d<l-1$, then the generic degree $d$ curve does not contain an $\mathbb{X}(l)$.

It is useful to recall that star configuration points have the same Hilbert function as generic points. More precisely, by [8, Lemma 7.8], we have

Lemma 2.4. Let $H F(\mathbb{X}(l), t)=\operatorname{dim}_{\mathbb{C}}\left(S / I_{\mathbb{X}(l)}\right)_{t}$ denote the Hilbert function of $S / I_{\mathbb{X}(l)}$. Then

$$
H F(\mathbb{X}(l), t)=\min \left\{\left(\begin{array}{c}
t+2 \\
2
\end{array}\right),\left(\begin{array}{l}
l \\
2
\end{array}\right)\right\} \text { for all } t \geq 0
$$

\section{An ASYMPtotic RESUlt}

In $\underline{3}$ it is shown that the generic degree $d$ plane curve contains a 0 -dimensional complete intersection scheme of type $(a, b)$ whenever $a, b \leq d$ (actually the result holds for any degree $d$ plane curve). Thus, arbitrarily large complete intersections can be found on generic plane curves of degree high enough. But the same does not hold for star configurations as shown by Theorem 3.1

Using the previous description of $I_{\mathbb{X}(l)}$ we introduce a quasi-projective variety parametrizing star configurations. Namely, we consider

$$
\mathcal{D}_{l} \subset \underbrace{\check{\mathbb{P}}^{2} \times \cdots \times \check{\mathbb{P}}^{2}}_{l}
$$

such that $\left(\ell_{1}, \ldots, \ell_{l}\right) \in \mathcal{D}_{l}$ if and only if no three of the lines $\ell_{i}$ pass through the same point; here, $\check{\mathbb{P}}^{2}$ denotes the dual projective space.

Theorem 3.1. If $l>5$ is an integer, then the generic degree $d$ plane curve does not contain any star configuration $\mathbb{X}(l)$.

Proof. It is enough to consider the case $d \geq l-1$ (see Corollary 2.3). Let $\mathbb{P} S_{d}$ be the space parametrizing degree $d$ plane curves and define the following incidence correspondence:

$$
\Sigma_{d, l}=\{(\mathcal{C}, \mathbb{X}(l)): \mathcal{C} \supset \mathbb{X}(l)\} \subset \mathbb{P} S_{d} \times \mathcal{D}_{l} .
$$

We also consider the natural projection maps

$$
\psi_{d, l}: \Sigma_{d, l} \longrightarrow \mathcal{D}_{l} \text { and } \phi_{d, l}: \Sigma_{d, l} \longrightarrow \mathbb{P} S_{d} .
$$

Clearly we have that $\phi_{d, l}$ is dominant if and only if Question 1.1 has an affirmative answer.

Since the fiber $\psi_{d, l}^{-1}(\mathbb{X}(l))$ is non-empty, the map $\psi_{d, l}$ is surjective. Hence, using a standard fibre dimension argument, we see that

$$
\operatorname{dim} \Sigma_{d, l}=\operatorname{dim} \mathcal{D}_{l}+\operatorname{dim} \psi_{d, l}^{-1}(\mathbb{X}(l))
$$

for a generic star configuration $\mathbb{X}(l)$, where $\operatorname{dim} \mathcal{D}_{l}=2 l$ and by Lemma 2.4

$$
\operatorname{dim} \psi_{d, l}^{-1}(\mathbb{X}(l))=\operatorname{dim}_{\mathbb{C}}\left(I_{\mathbb{X}(l)}\right)_{d}-1=\left(\begin{array}{c}
d+2 \\
2
\end{array}\right)-\left(\begin{array}{l}
l \\
2
\end{array}\right)-1 .
$$

Hence the map $\phi_{d, l}$ is dominant only if $\operatorname{dim} \Sigma_{d, l}-\operatorname{dim} \mathbb{P} S_{d} \geq 0$, and this is equivalent to

$$
2 l-\left(\begin{array}{l}
l \\
2
\end{array}\right)=\frac{l(5-l)}{2} \geq 0 .
$$

Thus the result is proved. 
By Lemma 2.1 and the above result, we only need to treat the cases $l=4$ and 5 . We postpone these cases to first rephrase Question 1.1 into an equivalent algebraic question.

\section{Restatement of Question 1.1}

We derive some technical results, moving our Question 1.1 back and forth between questions in algebra and questions in geometry. First, we notice the following trivial fact:

Lemma 4.1. Let $\{F=0\}$ be an equation of the degree $d$ curve $\mathcal{C} \subset \mathbb{P}^{2}$. Then $\mathcal{C}$ contains a star configuration $\mathbb{X}(l)$ only if

$$
F=\sum_{i=1}^{l} M_{i} \hat{L}_{i}
$$

where the forms $M_{i}$ have degree $d-l+1$ and the forms $\hat{L}_{i}$ are defined as $\hat{L}_{i}=$ $\prod_{j \neq i} L_{i}$ for some linear forms $L_{1}, \ldots, L_{l}$.

Hence, it is natural to perform the following geometric construction. We define a map of affine varieties

$$
\Phi_{d, l}: \underbrace{S_{1} \times \cdots \times S_{1}}_{l} \times \underbrace{S_{d-l+1} \times \cdots \times S_{d-l+1}}_{l} \longrightarrow S_{d}
$$

such that

$$
\Phi_{d, l}\left(L_{1}, \ldots, L_{l}, M_{1}, \ldots, M_{l}\right)=\sum_{i=1}^{l} M_{i} \hat{L}_{i} .
$$

We then rephrase our problem in terms of the map $\Phi_{d, l}$ :

Lemma 4.2. Let $d, l$ be nonnegative integers with $d \geq l-1$. Then the following are equivalent:

(i) Question 1.1 has an affirmative answer for $d$ and $l$.

(ii) The map $\Phi_{d, l}$ is a dominant map.

Proof. Lemma 4.1 proves that $(i)$ implies $(i i)$. To prove the other direction, it is enough to show that for a generic form $F$, the fibre $\Phi_{d, l}^{-1}(F)$ contains a set of $l$ linear forms defining a star configuration. More precisely, define $\Delta \subset S_{1} \times \cdots \times$ $S_{1} \times S_{d-l+1} \times \cdots \times S_{d-l+1}$ as follows:

$$
\Delta=\left\{\begin{array}{l|l}
\left(L_{1}, \ldots, L_{l}, M_{1}, \ldots, M_{l}\right) & \begin{array}{l}
\text { there exists } a \neq b \neq c \text { such that } \\
L_{a}, L_{b}, L_{c} \text { are linearly dependent }
\end{array}
\end{array}\right\} .
$$

Then we want to show that $\Phi_{d, l}^{-1}(F) \not \subset \Delta$.

We proceed by contradiction, assuming that the generic fibre of $\Phi_{d, l}$ is contained in $\Delta$. Then $\Delta$ would be a component of the domain of $\Phi_{d, l}$, thus a contradiction as the latter is an irreducible variety being the product of irreducible varieties.

Using the map $\Phi_{d, l}$ we can now translate Question 1.1 into an ideal theoretic question. 
Lemma 4.3. Let $d, l$ be non-negative integers. Consider generic forms $L_{1}, \ldots, L_{l} \in$ $S_{1}$ and $M_{1}, \ldots, M_{l} \in S_{d-l+1}$. Set

$$
\hat{L}_{i}=\prod_{j \neq i} L_{j} \text { and } \hat{L}_{i, j}=\prod_{h \neq i, h \neq j} L_{h}, \text { for } i \neq j .
$$

Also set

$$
\begin{aligned}
Q_{1} & =M_{2} \hat{L}_{1,2}+\cdots+M_{l} \hat{L}_{1, l}=\sum_{i \neq 1} M_{i} \hat{L}_{1, i}, \\
& \vdots \\
Q_{l} & =M_{1} \hat{L}_{l, 1}+\cdots+M_{l-1} \hat{L}_{l, l-1}=\sum_{i \neq l} M_{i} \hat{L}_{l, i} .
\end{aligned}
$$

With this notation, form the ideal

$$
I=\left(\hat{L}_{1}, \ldots, \hat{L}_{l}, Q_{1}, \ldots, Q_{l}\right) \subset S .
$$

Then the following are equivalent:

(i) Question 1.1 has an affirmative answer for $d$ and $l$.

(ii) $I_{d}=S_{d}$.

Proof. Using Lemma 4.2 we just have to show that $\Phi_{d, l}$ is a dominant map. In order to do this we will determine the tangent space to the image of $\Phi_{d, l}$ in a generic point $q=\Phi_{d, l}(p)$, where $p=\left(L_{1}, \ldots, L_{l}, M_{1}, \ldots, M_{l}\right)$ and we denote by $T_{q}$ this affine tangent space.

The elements of the tangent space $T_{q}$ are obtained as

$$
\left.\frac{d}{d t}\right|_{t=0} \Phi_{d, l}\left(L_{1}+t L_{1}^{\prime}, \ldots, L_{l}+t L_{l}^{\prime}, M_{1}+t M_{1}^{\prime}, \ldots, M_{l}+t M_{l}^{\prime}\right)
$$

when we vary the forms $L_{i}^{\prime} \in S_{1}$ and $M_{i}^{\prime} \in S_{d-l+1}$. By a direct computation we see that the elements of $T_{q}$ have the form

$$
\begin{gathered}
M_{1}^{\prime} \hat{L}_{1}+\cdots+M_{l}^{\prime} \hat{L}_{l}+L_{1}^{\prime}\left(M_{2} \hat{L}_{1,2}+\cdots+M_{l} \hat{L}_{1, l}\right) \\
+\cdots+L_{j}^{\prime}\left(M_{1} \hat{L}_{1, j}+\cdots+M_{l} \hat{L}_{j, l}\right)+\cdots+L_{l}^{\prime}\left(M_{1} \hat{L}_{1,2}+\cdots+M_{l-1} \hat{L}_{1, l-1}\right),
\end{gathered}
$$

where $\hat{L}_{i}=\prod_{j \neq i} L_{i}$ and $\hat{L}_{i, j}=\prod_{h \neq i, h \neq j} L_{h}$, for $i \neq j$. $T_{q}$.

Since the $L_{i}^{\prime} \in S_{1}$ and $M_{i}^{\prime} \in S_{d-l+1}$ can be chosen freely, we obtain that $I_{d}=$

Remark 4.4. Lemma 4.3 is an effective tool for giving a positive answer to each special issue of Question 1.1. Given $d$ and $l$, we construct the ideal $I$ as described by choosing forms $L_{i}$ and $M_{i}$. Then we compute $\operatorname{dim}_{\mathbb{C}} I_{d}$ using a computer algebra system, e.g., CoCoA. If $\operatorname{dim}_{\mathbb{C}} I_{d}=\operatorname{dim}_{\mathbb{C}} S_{d}$, by upper-semi-continuity of the dimension, we have proved that our question has an affirmative answer for these given $d$ and $l$. In fact, the dimension can decrease only on a proper closed subset. On the contrary, if $\operatorname{dim}_{\mathbb{C}} I_{d}<\operatorname{dim}_{\mathbb{C}} S_{d}$, we do not have any proof. Question 1.1 can both have a negative (for any choice of forms the inequality holds) or an affirmative answer (our choice of forms is too special and another choice will give an equality). But, if we choose our forms generic enough, we do have a strong indication that the answer to Question 1.1 should be negative. 
Example 4.5. We use CoCoA [5] to consider the example $(d, l)=(4,4)$.

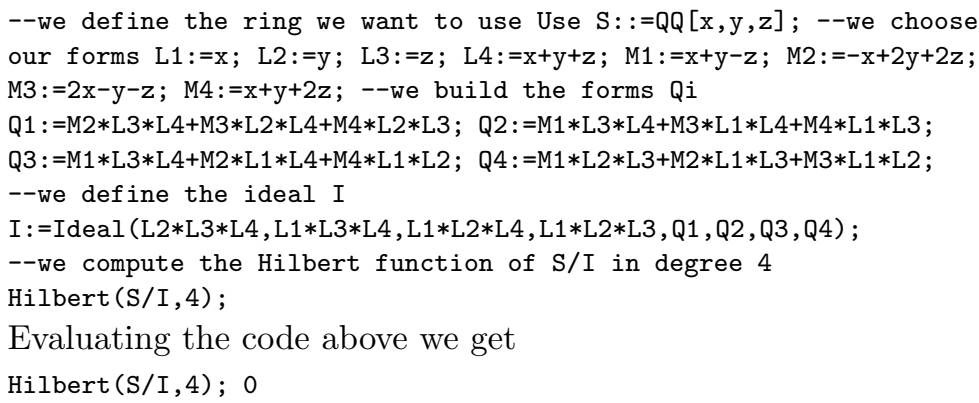

and this means that $\operatorname{dim}_{\mathbb{C}} S_{4}-\operatorname{dim}_{\mathbb{C}} I_{4}=0$. Hence we showed that the generic plane quartic contains an $\mathbb{X}(4)$.

\section{THE $l=4$ CASE}

In this section we consider Question 1.1 when $l=4$. We begin with a special instance of Question 1.1 that is, when $d=3$, since it has a nice geometric proof which takes advantage of the group structure on the curve.

Lemma 5.1. Let $\mathcal{C} \subset \mathbb{P}^{2}$ be a smooth cubic curve. Then there exists a star configuration $\mathbb{X}(4) \subset \mathcal{C}$.

Proof. We will produce an explicit geometric proof. However, a computational proof along the lines of Example 4.5 is possible. We will use the group law on $\mathcal{C}$ and hence we fix a point $p_{0} \in \mathcal{C}$ serving as identity. Then we choose a point $p_{2} \in \mathcal{C}$ such that $2 p_{2}=p_{0}$ and a generic point $p_{1} \in \mathcal{C}$. Consider the line joining $p_{1}$ and $p_{2}$ and let $p_{3}$ be the third intersection point with $\mathcal{C}$. Joining $p_{3}$ and $p_{0}$ and taking the third intersection we get the point $p_{1}+p_{2}$. Now join $p_{1}+p_{2}$ and $p_{2}$ and let $p_{4}$ be the third intersection point. Then joining $p_{4}$ and $p_{0}$ we get $p_{1}+2 p_{2}=p_{1}$. Hence, the points $p_{0}, p_{1}, p_{2}, p_{1}+p_{2}, p_{3}, p_{4} \in \mathcal{C}$ are a star configuration.

We now consider the general situation:

Theorem 5.2. Let $\mathcal{C} \subset \mathbb{P}^{2}$ be a generic degree $d \geq 3$ plane curve. Then there exists a star configuration $\mathbb{X}(4) \subset \mathcal{C}$.

Proof. The case $d=3$ is treated in Lemma 5.1 while for $d>3$ we will use Lemma 4.3 and the notation introduced therein. For $d=4$, we produced an explicit example in Example 4.5 where $I_{4}=S_{4}$, and this is enough to conclude by semi-continuity.

To deal with the general case $d \geq 5$, we use the structure of the coordinate ring of a star configuration. Given four generic linear forms $L_{1}, L_{2}, L_{3}, L_{4}$, we consider the star configuration they define, say $\mathbb{X}=\left\{p_{i, j}: 1 \leq i<j \leq 4\right\}$. The coordinate ring of $\mathbb{X}$ is

$$
A=\frac{S}{\left(\hat{L}_{1}, \ldots, \hat{L}_{4}\right)} .
$$

When $d \geq 2, \operatorname{dim}_{\mathbb{C}} A_{d}=6$. To prove that $I_{d}=S_{d}$, we want to find linear forms $N_{1}, \ldots, N_{6}$ such that the six forms $N_{i} Q_{i}$ are linearly independent in $A_{4}$. To check whether elements in $A$ are linearly independent it is enough to consider their 
evaluations at the points $p_{i, j}$. We now use the notation of Lemma 4.3 where the forms $M_{i}$ will be fixed later on in the proof. Consider the evaluation matrix

\begin{tabular}{c|cccc} 
& $Q_{1}$ & $Q_{2}$ & $Q_{3}$ & $Q_{4}$ \\
\hline & & & & \\
$p_{1,2}$ & $M_{2} L_{3} L_{4}$ & $M_{1} L_{3} L_{4}$ & 0 & 0 \\
$p_{1,3}$ & $M_{3} L_{2} L_{4}$ & 0 & $M_{1} L_{2} L_{4}$ & 0 \\
$p_{1,4}$ & $M_{4} L_{2} L_{3}$ & 0 & 0 & $M_{1} L_{2} L_{3}$ \\
$p_{2,3}$ & 0 & $M_{3} L_{1} L_{4}$ & $M_{2} L_{1} L_{4}$ & 0 \\
$p_{2,4}$ & 0 & $M_{4} L_{1} L_{3}$ & 0 & $M_{2} L_{1} L_{3}$ \\
$p_{3,4}$ & 0 & 0 & $M_{4} L_{1} L_{2}$ & $M_{3} L_{1} L_{2}$
\end{tabular}

obtained by evaluating each $Q_{i}$ at the points of the configuration where we denote, by abuse of notation, $M_{h} L_{i} L_{j}\left(p_{m, n}\right)$ with $M_{h} L_{i} L_{j}$. For example, since $Q_{2}=$ $M_{1} L_{3} L_{4}+M_{3} L_{1} L_{4}+M_{4} L_{1} L_{3}, Q_{2}\left(p_{2,3}\right)=M_{3} L_{1} L_{4}\left(p_{2,3}\right)$ because $L_{3}$ vanishes at $p_{2,3}$, while $Q_{2}\left(p_{1,4}\right)=0$ since $L_{4}$ and $L_{1}$ vanish at $p_{1,4}$. Now choose the forms $M_{i}$ with $\operatorname{deg} M_{i}=d-3 \geq 2$ so that

$$
M_{2}\left(p_{2,3}\right)=M_{3}\left(p_{3,4}\right)=M_{4}\left(P_{2,4}\right)=0
$$

and no other vanishing occurs at the points of the star configuration. Notice that this is possible as $\operatorname{dim}_{\mathbb{C}} A_{d}=6$ for $d \geq 2$. The matrix (5.1) can be represented as

\begin{tabular}{c|cccc} 
& $Q_{1}$ & $Q_{2}$ & $Q_{3}$ & $Q_{4}$ \\
\hline$p_{1,2}$ & $*$ & $*$ & 0 & 0 \\
$p_{1,3}$ & $*$ & 0 & $*$ & 0 \\
$p_{1,4}$ & $*$ & 0 & 0 & $*$ \\
$p_{2,3}$ & 0 & $*$ & 0 & 0 \\
$p_{2,4}$ & 0 & 0 & 0 & $*$ \\
$p_{3,4}$ & 0 & 0 & $*$ & 0
\end{tabular}

where $*$ denotes a non-zero scalar. As this matrix has rank four, then the forms $Q_{i}$ are linearly independent in $A$.

To represent $N_{i} Q_{i}$ in $A$ it is enough to multiply the $j$-th element of the $i$-th column of (5.1) by the evaluation of $N_{i}$ at $p_{j, i}$. Hence, if we choose the forms $N_{i}$ such that they do not vanish at any point of $\mathbb{X}$, the evaluation matrix of the $N_{i} Q_{i}$ has the same non-zero pattern as (5.2). Thus the forms $N_{1} Q_{1}, \ldots, N_{4} Q_{4}$ are linearly independent in $A$.

To complete the proof we need two more forms, and we choose $L_{1} Q_{3}$ and $L_{1} Q_{2}$ whose evaluation matrix at the points $p_{i, j}$ is

\begin{tabular}{c|cccccc} 
& $p_{1,2}$ & $p_{1,3}$ & $p_{1,4}$ & $p_{2,3}$ & $p_{2,4}$ & $p_{3,4}$ \\
\hline & & & & & & \\
$L_{1} Q_{3}$ & 0 & 0 & 0 & 0 & 0 & $*$ \\
$L_{1} Q_{2}$ & 0 & 0 & 0 & $*$ & 0 & 0
\end{tabular}

These rows are linearly independent with the columns of (5.2), completing the proof.

\section{THE $l=5$ CASE}

It remains to consider the case $l=5$. The case $l=5$ and $d=4$ was classically studied, a quartic containing a star configuration of ten points, that is, an $\mathbb{X}(5)$. 
For more details, see [12, 13, and for a modern treatment we refer to [14. In particular, we require the following property of Lüroth quartics:

Theorem (Theorem 11.4 of [14]). Lüroth quartics form a hypersurface of degree 54 in the space of plane quartics.

Corollary 6.1. Question 1.1 has a negative answer for $(d, l)=(4,5)$.

Proof. We notice that the previous theorem is enough to give a negative answer to our question for $l=5$ and $d=4$. In fact, a generic plane quartic is not a Lüroth quartic, and hence, no star configuration $\mathbb{X}(5)$ can be found on it.

For the remaining values $d>4$, we give an affirmative answer to Question 1.1

Theorem 6.2. The generic degree $d>4$ plane curve contains a star configuration $\mathbb{X}(5)$.

Proof. For the case $d=5$ we produce an explicit example and then we conclude by semi-continuity. For the general case, we produce a proof similar to Theorem [5.2.

For the $d=5$ case, let our linear forms be given by

$$
L_{1}=x, L_{2}=y, L_{3}=z, L_{4}=x+y+z, \text { and } L_{5}=2 x-3 y+5 z .
$$

To construct the polynomials $Q_{1}, \ldots, Q_{5}$, we make use of the following forms:

$$
\begin{gathered}
M_{1}=x+y-z, M_{2}=-x+2 y+2 z, M_{3}=2 x-y-z, \\
M_{4}=3 x+y-z, \text { and } M_{5}=4 x-4 y+3 z .
\end{gathered}
$$

Form the ideal $I$ as in Lemma 4.3. Using CoCoA [5] to compute $I_{5}$, we find that $I_{5}=S_{5}$.

For the $d \geq 6$ case, we use the notation of Lemma 4.3 and fix linear forms $L_{1}, \ldots, L_{5}$ and use $p_{i, j}$ to denote the ten points of $\mathbb{X}(5)$ defined by the linear forms $L_{i}$. We construct the evaluation table

\begin{tabular}{c|ccccc} 
& $Q_{1}$ & $Q_{2}$ & $Q_{3}$ & $Q_{4}$ & $Q_{5}$ \\
\hline & & & & & \\
$p_{1,2}$ & $M_{2} L_{3} L_{4} L_{5}$ & $M_{1} L_{3} L_{4} L_{5}$ & 0 & 0 & 0 \\
$p_{1,3}$ & $M_{3} L_{2} L_{4} L_{5}$ & 0 & $M_{1} L_{2} L_{4} L_{5}$ & 0 & 0 \\
$p_{1,4}$ & $M_{4} L_{2} L_{3} L_{5}$ & 0 & 0 & $M_{1} L_{2} L_{3} L_{5}$ & 0 \\
$p_{1,5}$ & $M_{5} L_{2} L_{3} L_{4}$ & 0 & 0 & 0 & $M_{1} L_{2} L_{3} L_{4}$ \\
$p_{2,3}$ & 0 & $M_{3} L_{1} L_{4} L_{5}$ & $M_{2} L_{1} L_{4} L_{5}$ & 0 & 0 \\
$p_{2,4}$ & 0 & $M_{4} L_{1} L_{3} L_{5}$ & 0 & $M_{2} L_{1} L_{3} L_{5}$ & 0 \\
$p_{2,5}$ & 0 & $M_{5} L_{1} L_{3} L_{4}$ & 0 & 0 & $M_{2} L_{1} L_{3} L_{4}$ \\
$p_{3,4}$ & 0 & 0 & $M_{4} L_{1} L_{2} L_{5}$ & $M_{3} L_{1} L_{2} L_{5}$ & 0 \\
$p_{3,5}$ & 0 & 0 & $M_{5} L_{1} L_{2} L_{4}$ & 0 & $M_{3} L_{1} L_{2} L_{4}$ \\
$p_{4,5}$ & 0 & 0 & 0 & $M_{5} L_{1} L_{2} L_{3}$ & $M_{4} L_{1} L_{2} L_{3}$
\end{tabular}

obtained by evaluating each $Q_{i}$ at the points of the configuration where we denote, by abuse of notation, $M_{h} L_{i} L_{j} L_{r}\left(p_{m, n}\right)$ with $M_{h} L_{i} L_{j} L_{r}$.

We work in the coordinate ring of the star configuration $A=\frac{S}{\left(\hat{L}_{1}, \ldots, \hat{L}_{5}\right)}$. Now consider

$$
L_{5} Q_{1}, L_{2} Q_{1}, L_{1} Q_{2}, L_{3} Q_{2}, L_{2} Q_{3}, L_{4} Q_{3}, L_{3} Q_{4}, L_{5} Q_{4}, L_{4} Q_{5}, L_{1} Q_{5}
$$


We want to show that they are linearly independent in $A$ for a generic choice of the forms $M_{i}, \operatorname{deg} M_{i}=d-4$. Again, it is enough to show this for a special choice of forms. We choose the forms $M_{i}$ in such a way that

$$
\begin{gathered}
M_{1}\left(p_{1,5}\right)=M_{4}\left(p_{3,4}\right)=M_{5}\left(p_{4,5}\right)=0, \\
M_{2}\left(p_{1,2}\right)=M_{2}\left(p_{2,5}\right)=M_{3}\left(p_{1,3}\right)=M_{3}\left(p_{2,3}\right)=0,
\end{gathered}
$$

and the following are non-zero:

$$
\begin{gathered}
M_{1}\left(p_{1,2}\right), M_{1}\left(p_{1,3}\right), M_{1}\left(p_{1,4}\right), M_{2}\left(p_{2,3}\right), M_{2}\left(p_{2,4}\right), M_{3}\left(p_{3,4}\right), M_{1}\left(p_{3,5}\right), \\
M_{4}\left(p_{1,4}\right), M_{4}\left(p_{2,4}\right), M_{4}\left(p_{4,5}\right), M_{5}\left(p_{1,5}\right), M_{5}\left(p_{2,5}\right), M_{5}\left(p_{3,5}\right) .
\end{gathered}
$$

Notice that these conditions can be satisfied because $\operatorname{deg} M_{i} \geq 2$ and $\operatorname{dim}_{\mathbb{C}} A_{2}=$ $6, \operatorname{dim}_{\mathbb{C}} A_{e}=10$ for $e \geq 3$. Then, evaluating the forms in (6.2) at the points $p_{i, j}$, we obtain the matrix

\begin{tabular}{c|cccccccccc} 
& $L_{5} Q_{1}$ & $L_{2} Q_{1}$ & $L_{1} Q_{2}$ & $L_{3} Q_{2}$ & $L_{2} Q_{3}$ & $L_{4} Q_{3}$ & $L_{3} Q_{4}$ & $L_{5} Q_{4}$ & $L_{4} Q_{5}$ & $L_{1} Q_{5}$ \\
\hline$p_{1,2}$ & 0 & 0 & 0 & $*$ & 0 & 0 & 0 & 0 & 0 & 0 \\
$p_{1,3}$ & 0 & 0 & 0 & 0 & $*$ & $*$ & 0 & 0 & 0 & 0 \\
$p_{1,4}$ & $*$ & $*$ & 0 & 0 & 0 & 0 & $*$ & $*$ & 0 & 0 \\
$p_{1,5}$ & 0 & $*$ & 0 & 0 & 0 & 0 & 0 & 0 & 0 & 0 \\
$p_{2,3}$ & 0 & 0 & 0 & 0 & 0 & $*$ & 0 & 0 & 0 & 0 \\
$p_{2,4}$ & 0 & 0 & $*$ & $*$ & 0 & 0 & $*$ & $*$ & 0 & 0 \\
$p_{2,5}$ & 0 & 0 & $*$ & $*$ & 0 & 0 & 0 & 0 & 0 & 0 \\
$p_{3,4}$ & 0 & 0 & 0 & 0 & 0 & 0 & 0 & $*$ & 0 & 0 \\
$p_{3,5}$ & 0 & 0 & 0 & 0 & $*$ & $*$ & 0 & 0 & $*$ & $*$ \\
$p_{4,5}$ & 0 & 0 & 0 & 0 & 0 & 0 & 0 & 0 & 0 & $*$
\end{tabular}

where $*$ denotes a non-zero scalar. One can verify that (6.3) has rank ten and hence the forms in (6.2) are linearly independent in $A$ and the thesis follows.

We can now prove our main theorem.

Theorem 6.3. Let $l \geq 2$. Then the generic degree $d$ plane curve contains a star configuration $\mathbb{X}(l)$ if and only if

(i) $l=2$ and $d \geq 1$ or

(ii) $l=3$ and $d \geq 2$ or

(iii) $l=4$ and $d \geq 3$ or

(iv) $l=5$ and $d \geq 5$.

Proof. Combine Lemmas 2.1 and 5.1. Corollaries 2.3 and 6.1. and Theorems 3.1. 5.2 , and 6.2

\section{FinAl REMARKS}

We conclude this paper with some additional comments.

7.1. A general algorithmic approach. Consider a family $\mathcal{F}$ of algebraic subschemes of $\mathbb{P}^{n}$ and ask the following question: does the generic hypersurface of degree $d$ in $\mathbb{P}^{n}$ contain an element of $\mathcal{F}$ ? 
In this generality, this question is probably infeasible. But, if the family $\mathcal{F}$ is well behaved enough, there is quite a lot that we can do. In fact, we can produce an algorithm yielding an answer. Of course, this is not saying that the answer will be obtained in a feasible amount of time. We sketch out the basics of this approach.

Suppose that for each element in $\mathcal{F}$ we know the structure of its defining ideal. Furthermore, suppose that we can describe a variety $X_{\mathcal{F}}$ whose general point corresponds to such an ideal. Then we can consider the product with a suitable affine space $X_{\mathcal{F}} \times \mathbb{A}^{n}$ and construct a map $\Phi: X_{\mathcal{F}} \times \mathbb{A}^{n} \rightarrow \mathbb{P} S_{d}$. The map $\Phi$ is such that $\operatorname{Im}(\Phi)$ is the variety of degree $d$ forms lying in an ideal of an element of $\mathcal{F}$.

The answer to our question is then affirmative if and only if $\Phi$ is a dominant map. Thus, we determine the tangent space to $\operatorname{Im}(\Phi)$ at a generic point $p$ obtaining a linear space $T_{p} \subseteq \mathbb{P} S_{d}$. Finally, computing the dimension of $T_{p}$ is just a rank computation. If $\operatorname{dim} T_{p}=\operatorname{dim} \mathbb{P} S_{d}$, we are done by upper-semi-continuity. Otherwise, there are two possibilities: either we picked a point $p$ that is too special or the answer to the question is negative and we need to find an explanation.

7.2. An inductive approach. We sketch out an alternative approach to prove Theorem 6.3 to deal with the cases $l=4, d \geq 3$ and $l=5, d \geq 5$. The idea is to proceed by induction on $d$ using as bases the case $(l, d)=(4,3)$ (e.g., proved using the group law) and the case $(l, d)=(5,5)$ (e.g., proved using our explicit approach). Then, knowing the case $(l, d)$, one proves the case $(l, d+1)$ by showing that the map $\phi_{d, l}$ has at least a fibre having its smallest component of the expected dimension (see the notation in the proof of Theorem 3.1). The crucial remark is that the expected dimension does not depend on $d$ and is actually $2 l-\left(\begin{array}{l}l \\ 2\end{array}\right)$. Let $F$ be a degree $d$ polynomial and consider $\phi_{d, l}^{-1}(F L)$ where $L$ is a general linear form. Then $F L$ has degree $d+1$. By induction one knows the dimension of the fibre of the map over $F$, which is the expected one, and then one has to show that this fibre is a component of the fibre over $F L$.

7.3. Further investigations. We think the kind of question we considered in this paper could be asked in a more general situation, and we see at least two promising generalizations. The first such natural generalization is to consider star configurations of sets of points in $\mathbb{P}^{n}$. In this context, one takes a family of $l \geq n$ general hyperplanes (instead of lines). Any $n$ such planes meet at a single point. The set of all such points is the generalized star configuration. Alternatively, one can consider star configurations of positive dimension. For example, take $l \geq 2$ general planes in $\mathbb{P}^{3}$ and consider the family of lines formed by the pairwise intersection of any two such planes. In ongoing work with E. Guardo, we are considering the first case.

\section{ACKNOWLEDGEMENTS}

This paper began when the first author visited the second author at Lakehead University. Theorem 6.3 was inspired by computer experiments using CoCoA 5 . The first author was partially supported by the Giovani Ricercatori grant 2008 of the Politecnico di Torino and by the GNSAGA part of INDAM (Italian Institute for Mathematics). Both authors acknowledge the financial support of NSERC. They also thank Brian Harbourne and Ciro Ciliberto for their input and feedback. 


\section{REFERENCES}

[1] C. Bocci, B. Harbourne, Comparing powers and symbolic powers of ideals. J. Algebraic Geom. 19 (2010), 399-417. MR2629595 (2011d:13021)

[2] E. Carlini, Codimension one decompositions and Chow varieties. In Projective varieties with unexpected properties, 67-79, Walter de Gruyter GmbH \& Co. KG, Berlin, 2005. MR 2202247 (2007f:14056)

[3] E. Carlini, L. Chiantini, A.V. Geramita, Complete intersections on general hypersurfaces. Mich. Math. J. 57 (2008), 121-136. MR.2492444 (2010b:14098)

[4] E. Carlini, L. Chiantini, A.V. Geramita, Complete intersection points on general surfaces in $\mathbb{P}^{3}$. Michigan Math. J. 59 (2010), 269-281. MR2677620

[5] CoCoATeam, CoCoA: a system for doing computations in commutative algebra. Available at http://cocoa.dima.unige.it.

[6] S. Cooper, B. Harbourne, Z. Teitler, Combinatorial bounds on Hilbert functions of fat points in the plane. J. Pure Appl. Algebra 215 (2011), 2165-2179.

[7] A.V. Geramita, B. Harbourne, J. Migliore. Personal communication.

[8] A.V. Geramita, J. Migliore, L. Sabourin. The first infinitesimal neighborhood of a linear configuration of points in $\mathbb{P}^{2}$. J. Algebra 298 (2006), no. 2, 563-611. MR2217628 (2007a:13016)

[9] A. Grothendieck. Cohomologie locale des faisceaux cohérents et théorèmes de Lefschetz locaux et globaux (SGA 2). Documents Mathématiques (Paris) [Mathematical Documents (Paris)], 4. Société Mathématique de France, Paris, 2005. Séminaire de Géométrie Algébrique du Bois Marie, 1962, Augmenté d'un exposé de Michèle Raynaud. [With an exposé by Michèle Raynaud], with a preface and edited by Yves Laszlo, revised reprint of the 1968 French original. MR.2171939 (2006f:14004)

[10] J. Harris. Algebraic geometry, A first course. Grad. Texts in Math., 133, Springer-Verlag, New York, 1992. MR.1182558 (93j:14001)

[11] S. Lefschetz. On certain numerical invariants of algebraic varieties with application to abelian varieties. Trans. Amer. Math. Soc. 22 (1921), no. 3, 327-406. MR.1501178

[12] J. Lüroth, Einige Eigenschaften einer gewissen Gattung von Curven vierter Ordnung. Math. Ann. 1 (1869), no. 1, 37-53. MR.1509610

[13] F. Morley, On the Lüroth quartic curve. Amer. J. Math. 41 (1919), no. 4, 279-282. MR.1506393

[14] G. Ottaviani, E. Sernesi, On the hypersurface of Luroth quartics. Michigan Math. J. 59 (2010), 365-394. MR2677627

[15] F. Severi. Una proprieta' delle forme algebriche prive di punti multipli. Rend. Accad. Lincei, II 15 (1906) 691-696.

[16] E. Szabó. Complete intersection subvarieties of general hypersurfaces. Pacific J. Math. 175 (1996), no. 1, 271-294. MR:1419484 (98g:14061)

Dipartimento di Matematica, Politecnico di Torino, Corso Duca degli Abruzzi 24, 10129 Turin, Italy

E-mail address: enrico.carlini@polito.it

Department of Mathematical Sciences, Lakehead University, Thunder Bay, Ontario, CANAdA, P7B 5E1

E-mail address: avantuyl@lakeheadu.ca 